\title{
The Relationship between the Retinal Nerve Fiber Layer Thickness and the Presence of Fragmented QRS Complexes in Patients with Hypertension
}

\author{
Tolga Doğan ${ }^{1}$, Mucahit Yetim ${ }^{1}$, Macit Kalçık ${ }^{1}$, Sucattin İlker Kocamış ${ }^{2}$, Oya Dönmez ${ }^{2}$, \\ Lütfü Bekar ${ }^{1}$
} ${ }^{1}$ Department of Cardiology, Hitit
University Faculty of Medicine,
Çorum, Turkey
${ }^{2}$ Department of Ophthalmology,
Hitit University Faculty of Medicine,
Çorum, Turkey

\section{Correspondence: \\ Macit Kalçık}

Address: Department of Cardiology, Hitit University Faculty of Medicine, Çorum, Turkey

Email: macitkalcik@yahoo.com

\begin{abstract}
Background: Fragmented QRS (fQRS) detected on electrocardiography (ECG) has been demonstrated to be a marker of myocardial fibrosis in hypertensive patients. Retinal microvascular abnormalities reflect cumulative microcirculatory damage resulting from hypertension and atherosclerosis. In this study, we aimed to evaluate the retinal nerve fiber layer (RNFL) thickness in hypertensive patients with and without fQRS complexes detected on ECG.

Methods: Consecutive patients who were diagnosed with hypertension were included in the study. ECG and transthoracic echocardiography were performed to all patients. fQRS was defined as additional $R^{\prime}$ wave or notching/splitting of S wave in two contiguous ECG leads. The RNFL thickness was measured by a spectral-domain optical coherence tomography device along a circle around the center of the optic disc.

Results: This observational case-control study enrolled 62 (42 female, mean age: $54.3 \pm 9.9$ years) hypertensive patients with fQRS and 42 (24 female, mean age: $53.1 \pm 11.8$ years) hypertensive control patients without fQRS between April 2017 and October 2017. There was no significant difference between groups regarding demagraphic, laboratory and echocardiographic parameters. In all retinal quadrants, the mean RNFL thickness did not differ significantly between patients with $\mathrm{fQRS}(+)$ and $\mathrm{fQRS}(-)$ group.
\end{abstract}

Conclusion: The presence of fQRS in the ECG had no significant effect on the RNFL thickness in hypertensive patients. Detection of fQRS may not be used as a useful non-invasive technique in the evaluation of atherosclerosis in hypertensive patients.

Keywords: echocardiography, electrocardiography, fragmented QRS, retinal nerve fiber layer, optical coherence tomography

\section{INTRODUCTION}

Hypertension is one of the most important causes of atherosclerosis. It is estimated that the prevalence will reach 1.5 billion in 2025 [1]. Uncontrolled hypertension is known to shorten life expectancy by approximately 5 years [2]. Left ventricular hypertrophy (LVH) secondary to hypertension is closely related to cardiovascular morbidity and mortality. [3]. Collagen accumulation occurs in interstitial tissue with myocyte hypertrophy developing in LVH. [4]. Accumulation of collagen also causes the interstitial and perivascular fibrosis. This leads to left ventricular dysfunction leading to decreased coronary reserve and deterioration of electrical activity [5].

Fragmented QRS (fQRS) is a depolarization disorder that can be easily detected on a 12-channel superficial electrocardiogram (ECG). It reflects the conduction delay that is caused by myocardial fibrotic tissue. Coronary artery disease and fQRS have been shown to be associated with myocardial scarring [6-9]. In a study with 500 subjects it was also stated that it may be superior to Q-wave in determining myocardial damage [10]. 
The retina is a unique tissue where arteries and veins can be directly evaluated. The potential of retinal microvascular abnormalities as markers for cardiovascular disease was noticed by Marcus Gunn who described the association of retinal microvascular features with hypertension, renal diseases and cerebrovascular diseases towards the end of the 19 th century $[11,12]$. Retinal microvascular abnormalities such as generalized arteriolar narrowing, focal arteriolar narrowing, arteriovenous crossover, and retinopathy reflect cumulative microcirculatory damage resulting from hypertension, atherosclerosis, aging, and other processes. Therefore, they are regarded as cardiovascular disease markers. [13,14].

The retinal nerve fiber layer (RNFL) is in the innermost layer of the retina and formed by retinal ganglion cell axons. The axonal damage is characterized by the thinning of RNFL. RNFL thickness can be affected by various neurodegenerative diseases $[15,16]$. In addition, some systemic diseases, such as diabetes mellitus, cause the thinning of RNFL [17-20]. The thickness of RNFL is now easily assessed by Optical Coherence Tomography (OCT) [21]. In a previous study, the effects of carotid intima media thickness on RNFL thickness in hypertensive patients were examined and it was assumed that the decrease in RNFL thickness might be related to atherosclerosis [22].

In this study, we aimed to evaluate the RNFL thickness by spectral-domain OCT (SD-OCT) device in hypertensive patients with and without fQRS complexes detected in ECG. Thus, the effect of fQRS on RNFL thickness was evaluated and it was investigated whether atherosclerosis can be detected by a new non-invasive method.

\section{MATERIALS AND METHODS}

This observational case-control study enrolled 62 (42 female, mean age: $54.3 \pm 9.9$ years) hypertensive patients with fQRS and 42 (24 female, mean age: 53.1 \pm 11.8 years) hypertensive control patients between April 2017 and October 2017. The study protochol was approved by the Local Ethics Committee. Patients with diabetes mellitus, coronary artery disease, glaucoma, macular edema, agerelated macular degeneration, high myopia and hypermetropia, anomalous optic history of ocular trauma, optic neuropathy, chronic kidney disease were excluded from the study. Office blood pressure measurements and transthoracic echocardiography were performed in each patient and blood samples for laboratory analyses were obtained.

All patients were evaluated by transthoracic echocardiography which was performed by two cardiologists by using Vivid S5 system (GE medical systems, Andover, MA, USA) device in left supine position and parasternal short axis scanning. Echocardiographic measurements of the cardiac dimensions were performed according to the guidelines set by the American Society of Echocardiography [23]. Basic measurements included left atrial dimeter, left ventricular posterior wall thickness, interventricular septal thickness, left ventricular end diastolic and end systolic diameters. Left ventricular ejection fraction was measured by using biplane Simpson's and Teicholz methods [24]. LVH was considered if left ventricle mass index was $>125 \mathrm{~g} / \mathrm{m}^{2}$ in males and $>110 \mathrm{~g} / \mathrm{m}^{2}$ in females [25]. Diagnosis of hypertension was based on antihypertensive drug use and /or systolic blood pressure higher than $135 \mathrm{mmHg}$ or diastolic blood pressure higher than $85 \mathrm{mmHg}$ on 24 hours ambulatory blood pressure measurement according to recommendations for the management of hypertension in the European Society of Cardiology guidelines [26].

The standard 12-lead ECGs were obtained at a paper speed of $25 \mathrm{~mm} / \mathrm{s}$, amplitude of $10 \mathrm{~mm} / \mathrm{mv}$, and a filter range 0.04 to $40 \mathrm{~Hz}$ from all patients. fQRS was defined as the presence of an additional $\mathrm{R}$ wave (R'), notching of the R or $\mathrm{S}$ wave, or the presence of fragmentation (more than one $R^{\prime}$ ) in two contiguous leads (Figure 1) [27]. Typical right or left bundle branch block pattern (QRS $\geq 120 \mathrm{~ms}$ ) and incomplete right bundle branch block were excluded. The ECGs were analyzed by 2 independent and experienced cardiologists, who were blinded to all data. Patients were then divided into two groups: fQRS(+) group and fQRS(-) group and all demographic, echocardiographic, laboratory and optic parameters were compared between the groups.

\section{Measurement of Retinal Nerve Fiber Layer Thickness}

Scans were acquired at $3.45-\mathrm{mm}$ diameter centered at the optic nerve head and the peripapillary RNFL thickness was quantified. Nasal, superior, inferior and average temporal, quadrant peripapillary RNFL thicknesses were obtained from the OCT with optic disc 200 cube scan protocol along a circle with a diameter of $3.46 \mathrm{~mm}$ around the center of the optic disc. OCT imaging was performed with the Cirrus Heidelberg SD-OCT (Carl Zeiss Meditec, Dublin, CA). OCT imaging was performed by the two ophthalmologists blinded to the patients.

\section{Statistical Analysis}

Statistical analyses were performed using IBM SPSS Statistics for Windows, Version 19.0. (IBM Corp. Armonk, NY). The variables were investigated using analytical methods (Kolmogorov-Smirnov/Shapiro-Wilk test) to determine whether or not they were approximately normally distributed. Descriptive statistics were reported as mean with standard deviation for continuous variables with normal distribution, median and $25^{\text {th }}-75^{\text {th }}$ percentile values for continuous variables without normal distribution, and frequencies with percentages for the categorical variables. Group comparisons for continuous variables were tested using Student $t$ test when data distribution was normal and using Mann-Whitney $U$ test when data distributions were not normal. Comparisons for categorical variables were evaluated by $\chi 2$ test. Significance level was accepted as $\mathrm{p}<0.05$ in all statistical analyses. 


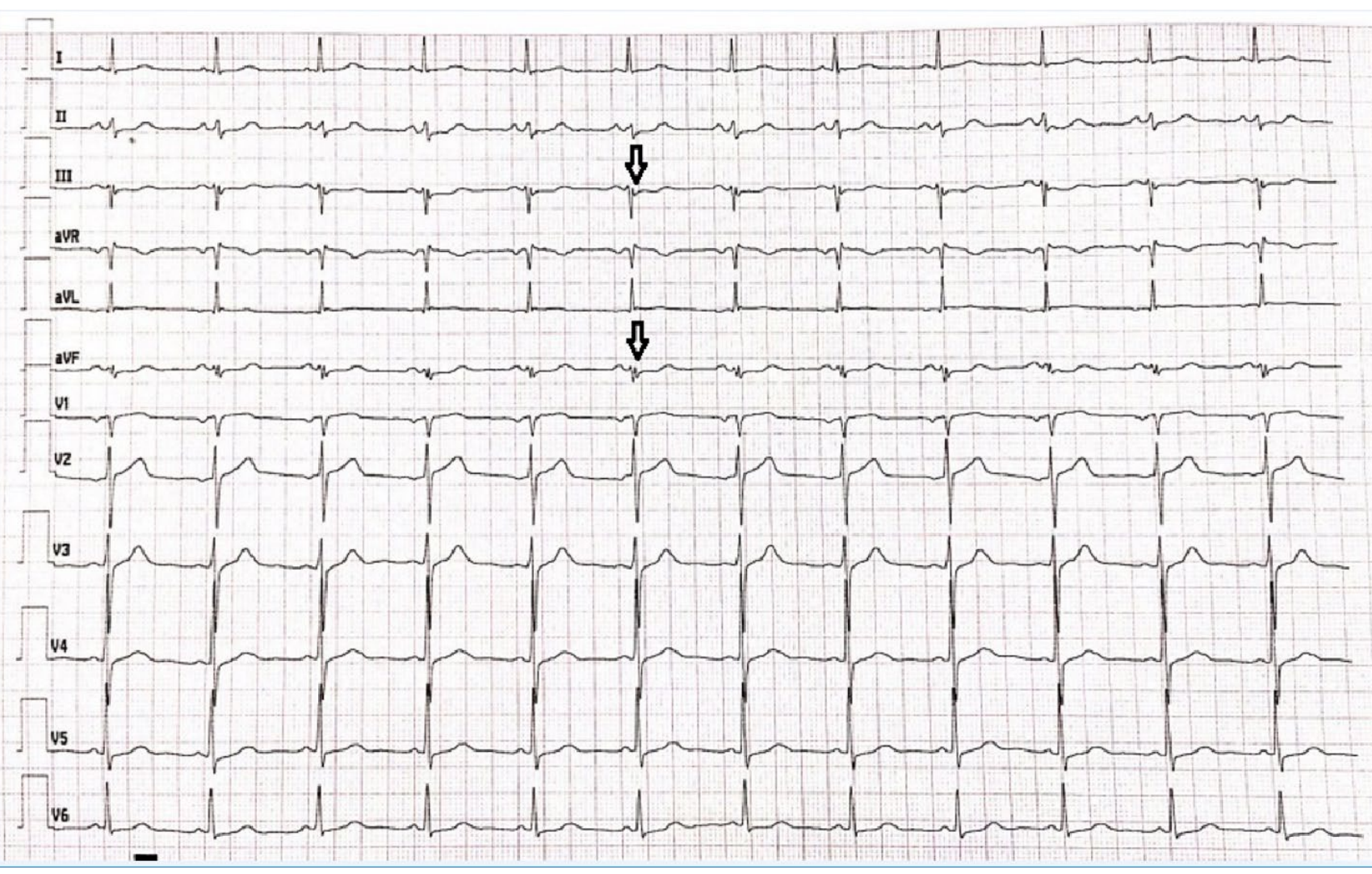

Figure 1. Electrocardiography revealing fragmented QRS complexes in two contiguous leads (III and aVF) in patient with hypertension

Table 1. Baseline clinical characteristics and used medications in the study population

\begin{tabular}{|c|c|c|c|}
\hline & fQRS (+) (group I) & fQRS (-) (group II) & $P$ value \\
\hline \multicolumn{4}{|l|}{ Demographical findings } \\
\hline Gender (male), n(\%) & $20(32.3)$ & $18(42.9)$ & 0.271 \\
\hline Age (years) & $54.3 \pm 9.9$ & $53.1 \pm 11.8$ & 0.586 \\
\hline BMI $\left(\mathrm{kg} / \mathrm{m}^{2}\right)$ & $30.1(25.9-33.12)$ & $29.7(26.75-32.4)$ & 0.596 \\
\hline ACEI, n(\%) & $23(37.1)$ & $21(50)$ & 0.191 \\
\hline ARB, $n(\%)$ & $29(46.8)$ & $17(40.5)$ & 0.526 \\
\hline CCB, $n(\%)$ & $23(37.1)$ & $10(23.8)$ & 0.153 \\
\hline Other Drugs, $\mathrm{n}(\%)$ & $25(40.3)$ & $14(33.3)$ & 0.47 \\
\hline SBP (mmHg) & $140(130-160)$ & $130(120-150)$ & 0.191 \\
\hline $\mathrm{DBP}(\mathrm{mmHg})$ & $80(80-92.5)$ & $80(80-88.75)$ & 0.242 \\
\hline
\end{tabular}

BMI: Body Mass Index, ACEI: Angiotensin Converting Enzyme Inhibitors ARB: Angiotensin Receptor Blockers, CCB: Calcium Channel Blocker, SBP: Systolic Blood Pressure, DPB: Diastolic Blood Pressure

\section{RESULTS}

Baseline clinical characterictics and medications used in fQRS(+) group and fQRS(-) group are summarized in Table 1. The results of laboratory and echocardiographic analyses were included in Table 2. All variables between patient and control groups were compared. There was no significant difference between groups according to age, gender, BMI, routine laboratory findings, echocardiographic measurements, blood pressures and medications used (Table 1-2). According to all quadrants, the mean RNFL thickness of the two groups is shown in Table 3. The RNFL thickness did not differ significantly between patients with fQRS $(+)$ and fQRS(-) group.

\section{DISCUSSION}

In our study, RNFL thickness was compared between hypertensive patients with and without fQRS detected on ECG and there was no significant difference between the groups.

The blood flow of RNFL is obtained from the retinal arteries, the choroidal arteries and the posterior ciliary artery. In a study published by Hayreh et al., the blood flow of the optic nerve is calculated by the ratio of perfusion 


\section{Retinal nerve fiber layer thickness and fragmented QRS}

Table 2. Baseline laboratory and echocardiographic findings in study groups

\begin{tabular}{|c|c|c|c|}
\hline & fQRS (+) (group I) & fQRS (-) (group II) & $P$ value \\
\hline \multicolumn{4}{|l|}{ Laboratory findings } \\
\hline WBC $\left(x 10^{3} / \mathrm{mL}\right)$ & $8.23 \pm 2.31$ & $8.36 \pm 2.14$ & 0.775 \\
\hline $\mathrm{Hb}(\mathrm{g} / \mathrm{dL})$ & $13.8(13.1-14.85)$ & $14.35(13.42-15.85)$ & 0.034 \\
\hline MPV (fL) & $10 \pm 1.17$ & $9.75 \pm 1.12$ & 0.287 \\
\hline $\mathrm{N} / \mathrm{L}$ ratio & $2.1(1.47-2.41)$ & $2.05(1.48-2.61)$ & 0.591 \\
\hline Urea $(\mathrm{g} / \mathrm{dL})$ & $27(33-23)$ & $28(24.13-33.8)$ & 0.379 \\
\hline Creatinine $(\mathrm{g} / \mathrm{dL})$ & $0.7(0.6-0.8)$ & $0.72(0.61-0.84)$ & 0.159 \\
\hline Glucose (mg/dL) & 97.5 (89-107.75) & $96(88.55-105.75)$ & 0.524 \\
\hline AST (U/L) & $21(17.75-26)$ & $20.5(18-23.75)$ & 0.501 \\
\hline $\operatorname{ALT}(U / L)$ & 21 (13.75-29.25) & $21(16-29.5)$ & 0.997 \\
\hline $\mathrm{Na}(\mathrm{mEq} / \mathrm{L})$ & $139.45 \pm 2.47$ & $139.6 \pm 2.17$ & 0.757 \\
\hline $\mathrm{K}(\mathrm{mEq} / \mathrm{L})$ & $4.28 \pm 0.34$ & $4.36 \pm 0.31$ & 0.244 \\
\hline Uric Acid (g/dL) & $5.15 \pm 01.27$ & $5.43 \pm 0.341 .41$ & 0.368 \\
\hline Total Bilirubin(U/L) & $0.4(0.32-0.57)$ & $0.37(0.34-0.61)$ & 0.532 \\
\hline GGT (mg/dL) & $21(18.5-42.5)$ & $20.5(14.75-66.75)$ & 0.925 \\
\hline Cholesterol (g/dL) & $197 \pm 35.22$ & $209.85 \pm 40.63$ & 0.093 \\
\hline $\mathrm{HDL}(\mathrm{g} / \mathrm{dL})$ & $48.31 \pm 12.37$ & $52 \pm 12.64$ & 0.145 \\
\hline $\mathrm{LDL}(\mathrm{g} / \mathrm{dL})$ & $119.39 \pm 27.85$ & $118.38 \pm 38.67$ & 0.878 \\
\hline Triglyceride (g/dL) & $133(101-194)$ & 154(113-191) & 0.256 \\
\hline \multicolumn{4}{|c|}{ Echocardiographic findings } \\
\hline LVEF (\%) & $60(60-60)$ & $60(60-61.25)$ & 0.268 \\
\hline LVESD (mm) & $30(29.5-35)$ & $30(28-30.5)$ & 0.028 \\
\hline LVEDD (mm) & $46(43-50)$ & $45(43-47)$ & 0.322 \\
\hline IVST (mm) & $11(11-12)$ & $11(11-12)$ & 0.073 \\
\hline PWT (mm) & $11(11-12)$ & $11(11-12)$ & 0.87 \\
\hline LAD (mm) & $38(36-40)$ & $38(36.75-40)$ & 0.584 \\
\hline$E(\mathrm{~cm} / \mathrm{s})$ & $0.6(0.5-0.7)$ & $0.7(0.5-0.9)$ & 0.272 \\
\hline$A(\mathrm{~cm} / \mathrm{s})$ & $1(0.8-1)$ & $0.95(0.7-1)$ & 0.253 \\
\hline
\end{tabular}

BNP:Brain natriuretic peptide,HDL: High Density Lipoprotein, LDL: Low Density, CRP :Serum Reactive Protein.Lipoprotein ,N/L: Neutrophil to lymphocyte ratio, LVEF: Left ventricular ejection fraction, LVESD: Left venticle end systolic volume, LVEDD: :Left venticle end diastolic volume, IVST: Interventricular septal thickness, PWT: Posterior wall thickness, LAD: Left atrial diameter,E: Atrial E wave, A: Atrial A wave

Table 3. Retinal nerve fiber layer thickness in patients with fQRS(+) and fQRS (-) groups

\begin{tabular}{cccc}
\hline Location & fQRS (+) (group I) & fQRS (-) (group II) & p value \\
\hline Average & $104.71 \pm 10.09$ & $104.79 \pm 10.94$ & 0.971 \\
\hline Superior Temporal & $139.42 \pm 22.86$ & $135.55 \pm 27.24$ & 0.89 \\
\hline Superior Nasal & $114.23 \pm 23.2$ & $115.33 \pm 23.62$ & 0.813 \\
\hline Inferior Temporal & $143(128.75-158.25)$ & $144.5(134-157)$ & 0.713 \\
\hline Inferior Nasal & $120.97 \pm 25.87$ & $119.57 \pm 25.34$ & 0.786 \\
\hline Temporal & $75.24 \pm 12.49$ & $77.64 \pm 14.57$ & 0.371 \\
\hline Nasal & $83.5(74-95)$ & $79(68-96.25)$ & 0.277
\end{tabular}

pressure to current resistance. Vascular changes in the arteries and arterioles may affect the blood flow resistance of the optic nerve [28]. Increased arterial stiffness and autonomic dysregulation due to chronic ischemic diseases such as aging process, atherosclerosis, hypertension and diabetes mellitus also lead to increased blood pressure changes leading to optic nerve ischemia $[29,30]$.

In a study by Sahin et al. using OCT, it has been shown that RNFL thickness of hypertensive patients are thinner than the normotensive patient group, especially in the superior and inferior quadrants [22]. It has been emphasized 
that the decrease in RNFL may be related to atherosclerosis. Another study by $\mathrm{Xu}$ et al in rhesus monkeys showed that localized RNFL defects were associated with stage 2 and 3 hypertension and that these localized RNFL defects could be used as a retinal marker in arterial hypertension [31]. Retinal blood flow is affected by ischemic conditions when exposed to chronic inflammatory processes such as malignant hypertension, uncontrolled diabetes mellitus. Frequent fluctuations in blood pressure during these processes lead to retinal ischemia.

The presence of myocardial fibrosis in hypertensive patients is associated with increased cardiovascular risk, because it paves the way for left ventricular dysfunction, arrhythmias, and sudden cardiac death [32,33]. fQRS indicates the conduction delay caused by the fibrous tissue. Fibrotic tissue slows the rate of electrical conduction leading to nonhomogeneous ventricular activation. This is observed as a notch in the QRS complex in the ECG. Although endomyocardial biopsy is the gold standard for diagnosing of myocardial fibrosis, many studies have demonstrated the association of myocardial fibrosis with fQRS. The prevalence of fQRS in healthy adults is 7.2\% [34]. Progressive fibrotic process in left ventricular hypertrophy requires a long time. Therefore, the reason for not having a significant difference in RNFL thickness between the two groups in our study may be that the chronic inflammatory process has not started or that this process is in the early stage. It may also be due to the fact that fQRS has a normal variation in the population in a considerable number of cases. Short duration of disease and possibly better retinal blood circulation autoregulation in young patients may be responsible for the survival of retinal ganglion cell axons. Moreover, the fact that optic ischemic retinopathy occurs with different mechanisms also affects results of our study. Large scaled cohort studies should be performed to clarify the relationship between fQRS and RNFL.

The present study has some limitations. First, it is a nonrandomized retrospective study which has been conducted with a quite small sample size in a single center. Second, only the patients with a QRS duration $<120 \mathrm{~ms}$ were included in the study. Including patients in the early-stage of hypertension with regulated blood pressures and middle age patients without diabetes mellitus may have effects on the study results.

\section{CONCLUSION}

In this study, the effect of the presence of fQRS on the RNFL thickness was investigated in hypertensive patients. The presence of fQRS on ECG had no significant effect on the RNFL thickness in hypertensive patients. Detection of fQRS may not be used as a useful non-invasive technique in the evaluation of atherosclerosis in hypertensive patients.

Declaration of interest: The authors report no conflicts of interest. Financial Disclosure: No financial support was received.

\section{REFERENCES}

1. Chobanian AV. Shattuck Lecture. The hypertension paradox-more uncontrolled disease despite improved therapy. N Engl J Med. 2009;361(9):878-87.

2. Franco OH, Peeters A, Bonneux L, de Laet C. Blood pressure in adulthood and life expectancy with cardiovascular disease in men and women. Hypertension 2005;46:280-6.

3. Ashton $\mathrm{N}$ : The eye in malignant hypertension. Trans Am Acad Ophthalmol Otolaryngol. 1972; 76:17-40.

4. Querejeta R, Varo N, Lopez B, Larman M, Artinano E, Etayo JC, Martinez Ubago JL, Gutierrez-Stampa M, Emparanza JI, Gil MJ, Monreal I, Mindan JP and Diez J. Serum carboxy-terminal propeptide of procollagen type I is a marker of myocardial fibrosis in hypertensive heart disease. Circulation. 2000;101:1729-35.

5. Kahan T, Bergfeldt L. Left ventricular hypertrophy in hypertension: its arrhythmogenic potential. Heart. 2005;91:250-6.

6. Basaran Y, Tigen K, Karaahmet T, et al. Fragmented QRS complexes are associated with cardiac fibrosis and significant intraventricular systolic dyssynchrony in nonischemic dilated cardiomyopathy patients with a narrow QRS interval. Echocardiography. 2011;28:62-8.

7. Das MK, Suradi H, Maskoun W, et al. Fragmented wide QRS on a 12-lead ECG: a sign of myocardial scar and poor prognosis. Circ Arrhythm Electrophysiol. 2008;1:258-68.

8. Park SJ, On YK, Kim JS, et al. Relation of fragmented QRS complex to right ventricular fibrosis detected by late gadolinium enhancement cardiac magnetic resonance in adults with repaired tetralogy of fallot. Am J Cardiol. 2012;109:110-5.

9. Homsi M, Alsayed L, Safadi B, et al. Fragmented QRS complexes on 12-lead ECG: a marker of cardiac sarcoidosis as detected by gadolinium cardiac magnetic resonance imaging. Ann Noninvasive Electrocardiol. 2009;14:319-26.

10. Das MK, Khan B, Jacob S, Kumar A, Mahenthiran J. Significance of a fragmented QRS complex versus a $Q$ wave in patients with coronary arter disease. Circulation. 2006;113:2495-501.

11. Gunn RM: On ophthalmoscopic evidence of general arteopathy: a review of existing classification systems and a sugrial disease. Trans Ophthalmol Soc UK 1898; 18:356-81.

12. Gunn RM: Opthalmocsopic evidence of (1) arterialchanges associated with chronic renal diseases and (2) of increased arterial tension. Trans Ophthalmol Soc UK 1892;12:124-5.

13. Ashton $\mathrm{N}$ : The eye in malignant hypertension. Trans Am Acad Ophthalmol Otolaryngol. 1972;76:17-40.

14. Ashton N, Peltier S, Garner A: Experimental hypertensive retinopathy in the monkey. Trans Ophthalmol Soc UK 1969;88:167-86. 
15. Pueyo V, Ara JR, Almarcegui C, Martin J, Güerri N, Garcia E et al. Subclinical atrophy of the retinal nerve fiberlayer in multiple sclerosis.Acta Ophthalmol. 2013;88:748-52.

16. Kirbas S, Turkyilmaz K,Anlar O,Tufekci A, Durmus M. Retinal nerve fiber layer thickness in patients with Alzheimer disease . J Neuro-Ophthalmol. 2013;33:5861.

17. Oshitari T, Hanawa K, Adachi-Usami E. Changes of macular and RNFL thicknesses measured by Stratus OCT in patients with early stage diabetes. Eye (Lond). 2009;23:884-9.

18. Sugimoto M, Sasoh M, Ido M, Wakitani $Y$, Takahashi C, Uji Y. Detection of early diabetic change with optical coherence tomography in type 2 diabetes mellitus patients without retinopathy. Ophthalmologica. 2005;219:379-85.

19. Park HY, Kim IT, Park CK .Early diabetic changes in the 25. nerve fi bre layer at the macula detected by spectral domain optical coherence tomography. Br J Ophthalmol. 2011;95:1223-8.

20. Shahidi AM, Sampson GP, Pritchard N, Edwards K, Vagenas D, Russell AW, et al. Retinal nerve fiber layer thinning associated with diabetic peripheral neuropathy. Diabet Med. 2012;29:e106-e111.

21. Kong M, Kwun Y, Sung J,Ham D and Song Y M. Association between systemic hypertension and macular thickness measured by optical coherence tomograph. Invest Ophthalmol Vis Sci. 2015;56:2144- 50.

22. Sahin O, Sahin S B, Ayaz T, Karadag Z, Turkyılmaz K, Aktas E, Bostan M. The impact of hypertension on retinal nerve fiber layer thickness and its association with carotid intima media thickness Blood Pressure, 2015; 24: 178-84.

23. Sahn DJ, DeMaria A, Kisslo J, et al. Recommendations regarding quantitation in M-mode echocardiography: results of a survey of echocardiographic measurements. Circulation 1978;58:1072.

24. Peter W Wood, Jonathan B Choy, Navin C Nanda, Harald Becher. Left Ventricular Ejection Fraction and Volumes: It Depends on the Imaging Method. Echocardiography. 2014 Jan;31(1):87-100.

25. Lang RM, Bierig M, Devereux RB, et al. American Society of Echocardiography's Nomenclature and
Standards Committee; Task Force on Chamber Quantification; American College of Cardiology Echocardiography Committee; American Heart Association; European Association of Echocardiography. European Society of Cardiology. Recommendations for chamber quantification. Eur J Echocardiogr. 2006;7:79-108.

26. Mancia G, De Backer G, Dominiczak A, et al. Guidelines for the management of arterial hypertension: the Task Force for the Management of Arterial Hypertension of the European Society of Hypertension (ESH) and of the European Society of Cardiology (ESC). Eur Heart J 2007;28:1462-536.

27. Pietrasik G, Zaręba W. QRS fragmentation: diagnostic and prognostic significance. Cardiol J. 2012;19(2):11421.

28. Hayreh SS. Ischemic optic neuropathy. Progress in Retina and Eye Research. 2009;28(1):34-62.

29. Stehouwer CD, Henry RM and Ferreira I. Arterial stiffness in diabetes and the metabolic syndrome: a pathway to cardiovascular disease. Diabetologia 2008;51:527-39.

30. Valensi P, Paries J and Attali JR; French Group for Research and Study of Diabetic Neuropathy. Cardiac autonomic neuropathy in diabetic patients: influence of diabetes duration, obesity, and microangiopathic complications - the French multicenter study. Metabolism 2003;52:815-820.

31. $\mathrm{Xu} \mathrm{L}$, Zhou JQ, Wang S, et al. Localized retinal nerve fiber layer defects and arterial hypertension. Am J Hypertens. 2013;26(4):511-7.

32. Kannel WB. Left ventricular hypertrophy as a risk factor in arterial hypertension. Eur Heart J 1992;13(Suppl D):82-8. 22.

33. Messerli FH, Grodzicki T. Hypertension, left ventricular hypertrophy, ventricular arrhythmias and sudden death. Eur Heart J 1992;13(Suppl D):66-9.

34. Bayramoğlu A, Taşolar H, Bektaş O, Yaman M, Kaya Y, Özbilen M, Kaya A. Association between metabolic syndrome and fragmented QRS complexes: Speckle tracking echocardiography study. J Electrocardiol. 2017;50(6):889-93. 